University of Wollongong

Research Online

Faculty of Engineering and Information

Faculty of Engineering and Information

Sciences - Papers: Part A

Sciences

$1-1-2015$

Continuous table acquisition MRI for radiotherapy treatment planning: distortion assessment with a new extended 3D volumetric phantom

Amy Walker

University of Wollongong, aw554@uowmail.edu.au

Gary Liney

University of Wollongong

Lois Holloway

University of Wollongong, loish@uow.edu.au

Jason Dowling

CSIRO

David Rivest-Henault

CSIRO

See next page for additional authors

Follow this and additional works at: https://ro.uow.edu.au/eispapers

Part of the Engineering Commons, and the Science and Technology Studies Commons

Research Online is the open access institutional repository for the University of Wollongong. For further information contact the UOW Library: research-pubs@uow.edu.au 


\title{
Continuous table acquisition MRI for radiotherapy treatment planning: distortion assessment with a new extended 3D volumetric phantom
}

\author{
Abstract \\ Purpose: Accurate geometry is required for radiotherapy treatment planning (RTP). When considering the \\ use of magnetic resonance imaging (MRI) for RTP, geometric distortions observed in the acquired images \\ should be considered. While scanner technology and vendor supplied correction algorithms provide some \\ correction, large distortions are still present in images, even when considering considerably smaller scan \\ lengths than those typically acquired with CT in conventional RTP. This study investigates MRI acquisition \\ with a moving table compared with static scans for potential geometric benefits for RTP. Methods: A full \\ field of view (FOV) phantom (diameter $500 \mathrm{~mm}$; length $513 \mathrm{~mm}$ ) was developed for measuring geometric \\ distortions in MR images over volumes pertinent to RTP. The phantom consisted of layers of refined \\ plastic within which vitamin E capsules were inserted. The phantom was scanned on CT to provide the \\ geometric gold standard and on MRI, with differences in capsule location determining the distortion. MRI \\ images were acquired with two techniques. For the first method, standard static table acquisitions were \\ considered. Both 2D and 3D acquisition techniques were investigated. With the second technique, images \\ were acquired with a moving table. The same sequence was acquired with a static table and then with \\ table speeds of $1.1 \mathrm{~mm} / \mathrm{s}$ and $2 \mathrm{~mm} / \mathrm{s}$. All of the MR images acquired were registered to the CT dataset \\ using a deformable B-spline registration with the resulting deformation fields providing the distortion \\ information for each acquisition. Results: MR images acquired with the moving table enabled imaging of \\ the whole phantom length while images acquired with a static table were only able to image $50 \%-70 \%$ of \\ the phantom length of $513 \mathrm{~mm}$. Maximum distortion values were reduced across a larger volume when \\ imaging with a moving table. Increased table speed resulted in a larger contribution of distortion from \\ gradient nonlinearities in the through-plane direction and an increased blurring of capsule images, \\ resulting in an apparent capsule volume increase by up to $170 \%$ in extreme axial FOV regions. Blurring \\ increased with table speed and in the central regions of the phantom, geometric distortion was less for \\ static table acquisitions compared to a table speed of $2 \mathrm{~mm} / \mathrm{s}$ over the same volume. Overall, the best \\ geometric accuracy was achieved with a table speed of $1.1 \mathrm{~mm} / \mathrm{s}$. Conclusions: The phantom designed \\ enables full FOV imaging for distortion assessment for the purposes of RTP. MRI acquisition with a \\ moving table extends the imaging volume in the $z$ direction with reduced distortions which could be \\ useful particularly if considering MR-only planning. If utilizing MR images to provide additional soft tissue \\ information to the planning CT, standard acquisition sequences over a smaller volume would avoid \\ introducing additional blurring or distortions from the through-plane table movement. \\ Disciplines \\ Engineering | Science and Technology Studies

\section{Publication Details} \\ Walker, A., Liney, G., Holloway, L., Dowling, J., Rivest-Henault, D. \& Metcalfe, P. (2015). Continuous table \\ acquisition MRI for radiotherapy treatment planning: Distortion assessment with a new extended 3D \\ volumetric phantom. Medical Physics, 42 (4), 1982-1991.
}

\section{Authors}

Amy Walker, Gary Liney, Lois Holloway, Jason Dowling, David Rivest-Henault, and Peter Metcalfe 
Continuous table acquisition MRI for radiotherapy treatment planning: distortion assessment with a new extended 3D volumetric phantom

Amy Walker $^{1,2}$, Gary Liney ${ }^{1,2,3}$, Lois Holloway ${ }^{1,2,3,4}$, Jason Dowling ${ }^{5}$, David Rivest-Henault ${ }^{5}$, Peter Metcalfe ${ }^{1,2}$ 5

Affiliations:

1. Centre for Medical Radiation Physics, University of Wollongong, Wollongong, NSW 2522, Australia

2. Liverpool and Macarthur Cancer Therapy Centres and Ingham Institute for Applied Medical Research,

10 Liverpool, NSW, Australia

3. South West Clinical School, University of New South Wales, Sydney, NSW, Australia

4. Institute of Medical Physics, School of Physics, University of Sydney, Sydney, NSW, Australia

5. Commonwealth Scientific and Industrial Research Organisation, Australian E-Health Research Centre,

QLD

15

*Corresponding Author: aw554@uowmail.edu.au

Key Words: MRI, geometric distortion, phantom, quality assurance, radiotherapy treatment planning, TimCT 


\section{Abstract:}

Purpose: Accurate geometry is required for radiotherapy treatment planning (RTP). When considering the use of magnetic resonance imaging (MRI) for RTP, geometric distortions observed in the acquired images should be considered. While scanner technology and vendor supplied correction algorithms provide some correction, large distortions are still present in images, even when considering considerably smaller scan lengths than those typically acquired with CT in conventional RTP. This study investigates MRI acquisition with a moving table compared with static scans for potential geometric benefits for RTP.

Methods: A full field of view (FOV) phantom (diameter $500 \mathrm{~mm}$; length $513 \mathrm{~mm}$ ) was developed for measuring geometric distortions in MR images over volumes pertinent to RTP. The phantom consisted of layers of refined plastic within which vitamin E capsules were inserted. The phantom was scanned on CT to provide the geometric gold standard and on MRI, with differences in capsule location determining the distortion. MRI images were acquired with two techniques. For the first method, standard static table acquisitions were considered. Both 2D and 3D acquisition techniques were investigated. With the second technique, images were acquired with a moving table. The same sequence was acquired with a static table and then with table speeds of $1.1 \mathrm{~mm} / \mathrm{s}$ and $2 \mathrm{~mm} / \mathrm{s}$. All of the MR images acquired were registered to the CT dataset using a deformable b-spline registration with the resulting deformation fields providing the distortion information for each acquisition.

Results: MR images acquired with the moving table enabled imaging of the whole phantom length while images acquired with a static table were only able to image $50-70 \%$ of the phantom length of $513 \mathrm{~mm}$. Maximum distortion values were reduced across a larger volume when imaging with a moving table. Increased table speed resulted in a larger contribution of distortion from gradient nonlinearities in the through plane direction and an increased blurring of capsule images, resulting in an apparent capsule volume increase by up to $170 \%$ in extreme axial FOV regions. Blurring increased with table speed and in the central regions of the phantom, geometric distortion was less for static table acquisitions compared to a table speed of $2 \mathrm{~mm} / \mathrm{s}$ over the same volume. Overall, the best geometric accuracy was achieved with a table speed of $1.1 \mathrm{~mm} / \mathrm{s}$. 
45 Conclusions: The phantom designed enables full FOV imaging for distortion assessment for the purposes of RTP. MRI acquisition with a moving table extends the imaging volume in the $z$ direction with reduced distortions which could be useful particularly if considering MR-only planning. If utilising MR images to provide additional soft tissue information to the planning $\mathrm{CT}$, standard acquisition sequences over a smaller volume would avoid introducing additional blurring or distortions from the through plane table movement. 


\section{INTRODUCTION}

The radiotherapy treatment planning (RTP) process requires accurate patient geometry. It is fundamental for ensuring the dose planned is delivered to the tumour region whilst limiting dose to organs at risk (OAR). With an increase in the use of magnetic resonance imaging (MRI) for RTP purposes ${ }^{1}$, assessing geometric accuracy is important, particularly when considering the potential for MRI-only planning.

System related geometric distortions in MRI are created by non-uniformity of the main magnetic field $\left(B_{0}\right)$ and nonlinearities in the gradient fields. Distortions in MR images have decreased in magnitude with improvements in technology. Most modern scanners allow for shimming and on or off line application of vendor correction algorithms to decrease the gradient nonlinearity and some $B_{0}$ field inhomogeneity effects. While vendor supplied correction algorithms provide a reduction, residual distortions still remain in various regions of the scanner ${ }^{2-4}$.

Clinical MRI scanner performance is optimised around the isocenter of the scanner, with gradient linearity and $\mathrm{B}_{0}$ field homogeneity decreasing with increased distance from isocenter. Distortion magnitude therefore increases radially outwards, even with the application of correction algorithms ${ }^{3}$. Improvements in scanner and coil technology have resulted in the ability to image anatomy in regions at larger distances from isocenter than previously achievable, where distortion considerations become more imperative. The maximum diagnostic imaging field of view (FOV) is typically $40-50 \mathrm{~cm}$ in all imaging planes. For RTP, the loss in geometric accuracy with increasing distance from isocenter is likely to considerably reduce the maximum FOV. Geometric accuracy of within $2 \mathrm{~mm}$ is observed for RTP imaging and treatment $\mathrm{QA}^{5}$, with MRI distortions greater than this requiring consideration.

When utilising MRI for RTP, the required imaged anatomy depends on its application, whether acquired for use in conjunction with $\mathrm{CT}$ or for the purposes of MR-only planning. In some cases, registering MRI to CT may warrant smaller FOV coverage (e.g. prostate). In such cases images may only need to encompass the anatomy of interest for contouring and registration purposes. Other treatments (such as head and neck) may require a longer FOV which covers all of the patient anatomy both superiorly and inferiorly, as well as the patient contour. If considering MR-only planning, a larger axial FOV coverage 
would be required to ensure the patient skin surface is included for the required dosimetric calculations. For the purposes of RTP, the geometrical accuracy requirement over the scan length ( $x, y$ and $z$ coverage) is more stringent than that required for routine diagnostic imaging. Spatial distortion assessment is a vital part of MRI quality assurance (QA) for RTP in addition to other specific QA required for RTP (e.g. surface coil intensity correction ${ }^{6}$, laser alignment, etc.).

Modern clinical MRI scanners have moved towards wider and shorter bore designs to improve patient comfort. Magnetic field strength may also be increased from $1.5 \mathrm{~T}$ to $3.0 \mathrm{~T}$, allowing faster imaging with improved imaging quality ${ }^{7}$. These features can make the optimisation of scanners more challenging, consequently increasing the geometric distortions. More scanners have moved away from spherically optimised to cylindrically optimised magnets to account for these features and to ensure the optimisation is more suited to the shape of the patients. All of these scanner designs have characteristic uniformity and linearity distortions. The AAPM report 100 for $\mathrm{MR} \mathrm{QA}^{8}$ recommends the geometric accuracy of clinical MRI scanners should be determined. A number of studies have investigated this on MRI scanners for the purposes of RTP ${ }^{4,9-11}$. Comprehensive distortion mapping of each system is recommended to determine the tolerances and constraints to minimise MRI distortion impact for RTP or provide data for a correction scheme where appropriate.

On short bore systems, image acquisition with a moving table is one approach to increase the scan length in the $z$ direction. This allows images to be acquired close to the scanner isocenter, where $B_{0}$ and gradient performance is optimised. Potentially, this could reduce the severe distortion effects at the edge of scans and improve the usable imaging range superiorly and inferiorly for RTP. Images can be acquired close to the isocenter at different table positions over a limited scan stitched together ${ }^{12,13}$. A more recent approach involved image acquisition with the table moving continuously through the scanner at a constant velocity (sliding multislice $(\mathrm{SMS}))^{14-16}$. Moving-table acquisitions have been investigated in detail in magnetic resonance angiography (MRA) and whole body $\mathrm{MRI}^{15,16}$, as well as for screening and staging in oncology ${ }^{12,17}$. Investigations into its utilisation in radiotherapy is limited ${ }^{13}$. While the SMS technique can reduce the magnitude of distortions present in an image for a larger scan length, it does not completely 
remove distortions. The technique is designed to reduce the distortion components from the $z$ axis with no additional benefit in the axial plane. One angiography study using SMS ${ }^{18}$ demonstrated additional distortion effects and blurring in all 3 imaging planes when utilising this acquisition technique.

Many commercially developed phantoms for distortion assessment are designed for diagnostic MRI QA, not for radiotherapy purposes. Multiple imaging parameters such as geometric distortion, uniformity, resolution, chemical shift, signal to noise (SNR) and ghosting can be tested within one phantom. As a result, each phantom compartment is quite small. A number of distortion studies have been conducted utilising such phantoms ${ }^{19-22}$. These phantoms can provide distortion analysis over a restricted FOV before requiring manual repositioning of the phantom ${ }^{23}$ over a limited number of points. Our previous work ${ }^{3}$ has shown limitations of one such commercial diagnostic phantom to adequately determine distortion across all 3 imaging planes, hence missing regions where patient anatomy may need to be imaged for RTP. A number of studies have developed their own distortion phantoms, for specific applications ${ }^{24-28}$. In this study, we present a phantom for characterising whole FOV geometric distortion on a MRI scanner. The new 3D-phantom is novel due to its extension in length and the usage of numerous offset Vitamin E capsules rather than rods for through plane distortion assessment. Investigations were made to assess the performance of a moving table image acquisition option to determine potential benefit of this technique for RTP. Comparisons were made to conventional static table 2D and 3D image acquisition sequences. A sound understanding of the geometrical limitations for static and moving table acquisitions is essential for investigations into MRI acquisition techniques for utilisation in RTP. This study investigates distortions from systematic factors with different acquisition techniques: a) static Vs moving table acquisition, b) variations in moving table speed, and c) vendor supplied 2D and 3D correction algorithms. Other distortion considerations including patient effects (susceptibility and chemical shift) on distortion as well as the impact of geometric distortion on inhomogeneity in MR images are presented elsewhere ${ }^{2,11,29-}$ 31.

\section{MATERIALS AND METHODS}


The phantom (Fig. 1) consisted of layers of flat sheet panel polyethylene and polypropylene (Dotmar Uniboard Eco) as the housing material. Vitamin E capsules were inserted throughout to provide the MRI signal. The phantom was cylindrical in shape with a flat bottom for stability giving a diameter of $500 \mathrm{~mm}$ and height of $375 \mathrm{~mm}$. The length of the phantom was $513 \mathrm{~mm}$, exceeding the limits of the standard imaging capabilities of the MRI scanner utilised, quoted as $z=450 \mathrm{~mm}$.

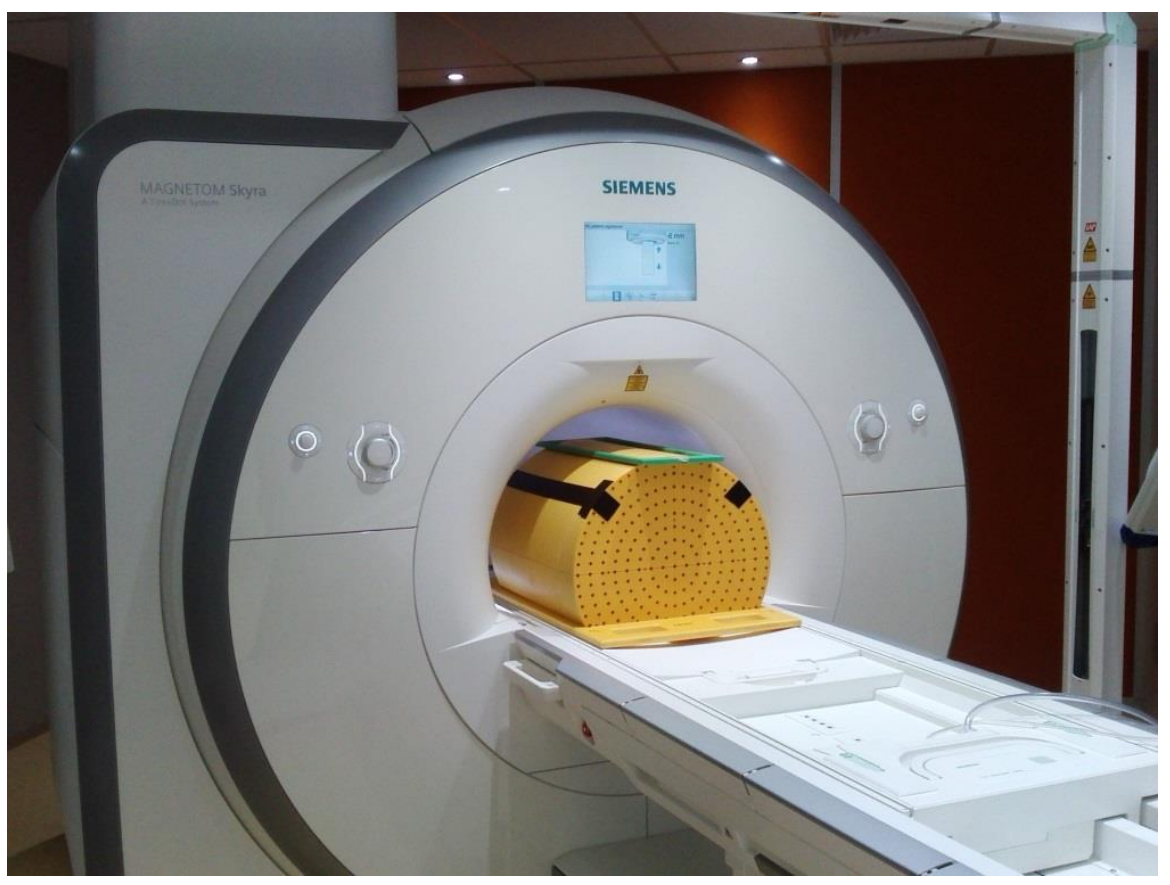

Fig. 1: Photograph of the distortion phantom positioned in a $70 \mathrm{~cm}$ wide bore 3 T Siemens Skyra 227 capsules and 13 layers containing 204 capsules. This resulted in a total of 5830 capsules inserted into the phantom. Holes $8 \mathrm{~mm}$ in diameter were drilled through each layer to ensure tight fit of the vitamin $\mathrm{E}$ capsules to reduce any air gaps. A cylindrical capsule shape (diameter $8 \mathrm{~mm}$; length $19 \mathrm{~mm}$ ) was chosen to minimise susceptibility effects from the capsules themselves ${ }^{32}$. being defined in polar coordinates. This offset of grid position enabled observation of through plane 
distortions. In the central phantom compartment, 2 axially centralised capsules were removed from the phantom in order to enable localisation of the central phantom region within the acquired images.

The phantom material had a density of $\sim 0.7 \mathrm{~g} / \mathrm{cm}^{3}$ to reduce the overall weight, with the total $\mathrm{mm} \times 500 \mathrm{~mm}$ ) enabled steady transfer of the phantom between CT and MRI. A central section of the base tray (dimensions of $513 \mathrm{~mm} \times 445 \mathrm{~mm}$, corresponding to the phantom base) was pitted, leaving a thickness of $9 \mathrm{~mm}$. Within this, the phantom body was placed and the base section held together rigidly. Ten plugs made from the same Uniboard material with length of $19 \mathrm{~mm}$ and a diameter of $5 \mathrm{~mm}$ were positioned between every layer to aid in holding the phantom together.

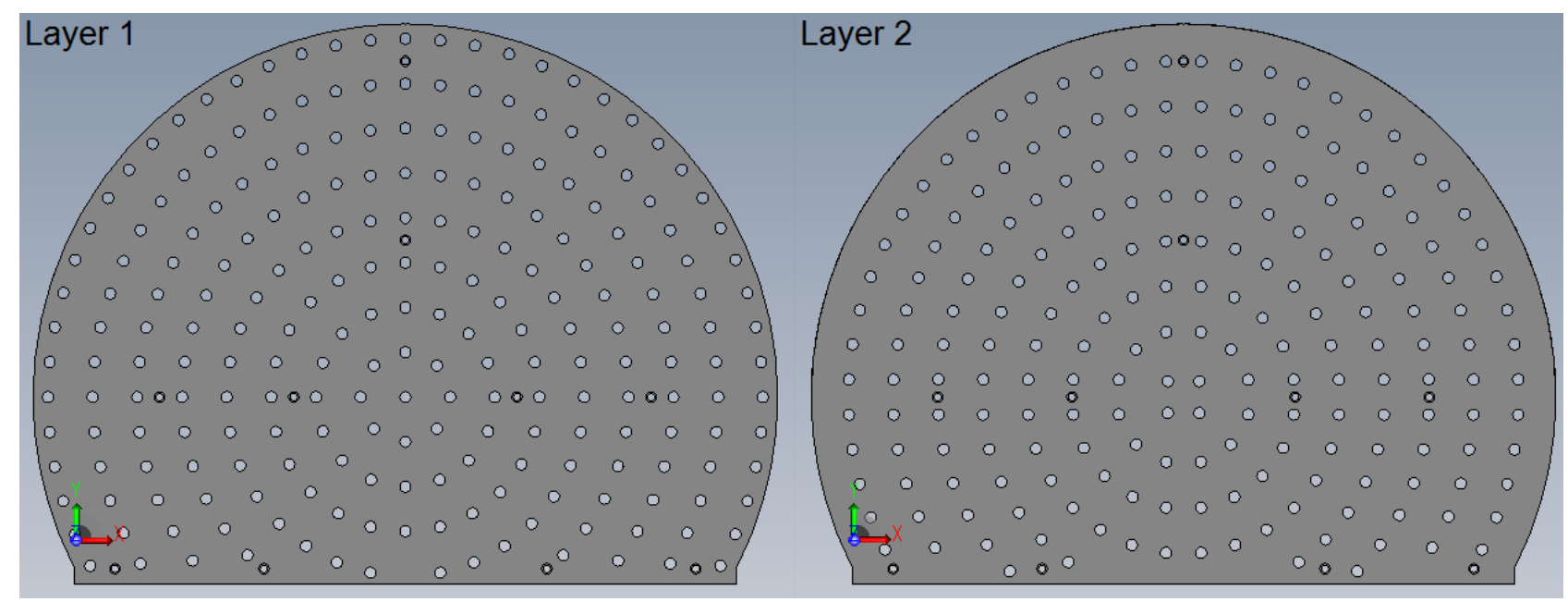

Fig. 2: Alternating layers in the phantom. Note the offset grid pattern between each layer for 3D point localisation. This ensures distorted points don't overlap with capsules in adjacent layers, aiding in through plane distortion assessment

\section{II.B. CT}

To validate the geometry and determine the location of the capsules within the phantom, a CT image provided the baseline geometric 'gold standard'. The phantom was scanned on an $85 \mathrm{~cm}$ bore, Brilliance CT big bore oncology CT scanner (Philips Healthcare). All CT images were acquired with a FOV of $500 \mathrm{~mm} \times 500 \mathrm{~mm}$ (512 $\times 512$ image resolution) with a scan length greater than $514 \mathrm{~mm}$ to ensure the 
whole phantom volume was imaged. Scans were acquired with a slice thickness of $1 \mathrm{~mm}$ so that the CT images could be used as a reference to analyse MR images of any slice thickness larger than $1 \mathrm{~mm}$.

MRI scans of the phantom were performed on a Siemens $70 \mathrm{~cm}$ bore Magnetom Skyra 3.0 T (Siemens Healthcare) (Fig. 1). The manufacturer quotes the gradient linearity as an average deviation equal to $0.2,0.3,0.2 \%(x, y, z \%)$ over $30 \mathrm{~cm}$ and $0.4,0.5,0.3 \%$ over $50 \mathrm{~cm}$. The magnet is cylindrically optimised, designed to achieve a homogeneous volume 1.5 times that of an ellipsoid system. Guaranteed homogeneity of the magnet over a $50 \mathrm{~cm} \times 50 \mathrm{~cm} \times 45 \mathrm{~cm}$ is quoted as less than 4 parts per million (ppm). All scans performed underwent standard tune-up $B_{0}$ shimming localised to the imaging volume.

For this study, 3 different acquisition methods were investigated:

1. Standard 2D image acquisition

2. 3D volume image acquisition

3. Continuously moving table acquisition: TimCT (Total Imaging Matrix, Continuous Table)

For each image acquisition sequence, 2 images of the phantom were obtained. The frequency encode directions between these 2 datasets were rotated by $180^{\circ}$ to enable separation of $B_{0}$ and gradient distortion components during the image analysis process ${ }^{33,34}$. A bandwidth of at least $440 \mathrm{~Hz} /$ pixel was implemented for each sequence as would be used for RT planning protocols ${ }^{7}$ to minimise susceptibility and chemical shift. Vendor 2D or 3D correction algorithms were applied during image acquisition as available. Scanner gradient nonlinearities are stored in the system as spherical harmonic coefficients, enabling the gradient field to be derived at any location within the magnet. The coefficients are used to calculate the gradient values for the scanner which are then applied to correct each voxel position and signal intensity accordingly throughout the imaged volume. The $2 \mathrm{D}$ correction provides only an in-plane correction to the distortion while the 3D correction accounts for through-plane distortion as well. These correction algorithms provide reproducible results within $0.5 \mathrm{~mm}$. Distortions were reported at the centre of each 
capsule as visualised on each slice within each dataset. Based on acceptance criteria for RTP imaging and treatment $\mathrm{QA}^{5}$, distortion values larger than $2 \mathrm{~mm}$ were considered to be significant.

\section{II.C.1. 2D and 3D acquisition sequences}

The imaging parameters for the static $2 \mathrm{D}$ and 3D image acquisitions are outlined in table 1 . For the 2D image acquisition, a standard spin echo (SE) sequence was used. A steady state gradient echo (GRE) sequence (CISS - constructive interface in steady state) provided the best image quality for the 3D acquisition of the phantom and was consequently investigated. The maximum scan lengths achievable for the given slice thickness and coil capabilities for each sequence was acquired. The phantom centre was aligned to the scanner isocenter. For these scans 2D correction was available as part of the imaging protocol whereas 3D correction was performed retrospectively on the scanner console.

Table 1: Acquisition parameters of standard imaging sequences

\begin{tabular}{cccccc}
\hline Sequence & $\begin{array}{c}\text { Slice thickness } \\
(\mathrm{mm})\end{array}$ & TE/TR $(\mathrm{ms})$ & $\begin{array}{c}\text { Scan length } \\
(\mathrm{mm})\end{array}$ & $\begin{array}{c}\text { Pixel BW } \\
(\mathrm{Hz} / \mathrm{pix})\end{array}$ & $\begin{array}{c}\text { Resolution } \\
\text { (pixels) }\end{array}$ \\
\hline \hline SE & 2 & $12 / 2760$ & 252 & 445 & $320 \times 320$ \\
$\mathrm{SE}$ & 3 & $12 / 2760$ & 368 & 445 & $320 \times 320$ \\
$\mathrm{CISS}$ & 2 & $3.15 / 6.84$ & 353 & 460 & $320 \times 320$ \\
\hline
\end{tabular}

\section{II.C.2. TimCT - extended FOV}

The phantom was placed on the scanner and covered with two 18 channel body coils for imaging the whole phantom volume with TimCT. The table was then moved into the scanner so that one end of the phantom was located at the scanner isocenter and the whole phantom could then be moved through the bore. All images were T1 in-phase Dixon spoiled GRE sequences acquired with $5 \mathrm{~mm}$ slice thickness. Table 2 shows the imaging parameters for different table speeds. The slab length refers to the scan length being imaged as the table moved and was dependent on the table speed selected. After images were acquired 
with the TimCT option selected, the phantom was setup with the phantom centre aligned to the scanner isocenter. The same sequence was then acquired with static table acquisition for comparison. These scans were all acquired with the vendor 3D correction algorithm applied.

Table 2: Acquisition parameters of the TimCT sequence and the corresponding sequence without the TimCT option utilised

\begin{tabular}{ccccccc}
\hline Sequence & $\begin{array}{c}\text { TE/TR } \\
(\mathrm{ms})\end{array}$ & $\begin{array}{c}\text { Scan length } \\
(\mathrm{mm})\end{array}$ & $\begin{array}{c}\text { Pixel BW } \\
(\mathrm{Hz} / \mathrm{pix})\end{array}$ & $\begin{array}{c}\text { Resolution } \\
(\text { pixels })\end{array}$ & $\begin{array}{c}\text { Table speed } \\
(\mathrm{mm} / \mathrm{s})\end{array}$ & $\begin{array}{c}\text { Slab length } \\
(\mathrm{mm})\end{array}$ \\
\hline Spoiled GRE (TimCT) & $1.47 / 190$ & 540.3 & 610 & $512 \times 512$ & 1.1 & 60 \\
$\begin{array}{c}\text { Spoiled GRE (TimCT) } \\
\begin{array}{c}\text { Spoiled GRE (Non } \\
\text { TimCT) }\end{array}\end{array}$ & $1.47 / 190$ & 550.5 & 610 & $512 \times 512$ & 2 & 110 \\
\hline
\end{tabular}

\section{II.D.1.Image registration:}

Three dimensional quantification of the geometric distortion was obtained by rigid and deformable registration between the $\mathrm{CT}$ and $\mathrm{MR}$ images. A robust inverse-consistent registration algorithm was used to rigidly align the CT and MR images within MILXView ${ }^{35}$. The algorithm handled the position and orientation information contained in the image header and provided a fully automatic result. All MR images acquired were rigidly registered to the $\mathrm{CT}$ with $1 \mathrm{~mm}$ slice thickness, ensuring all datasets were in the same frame of reference. The MR images then matched the $0.98 \times 0.98 \times 1 \mathrm{~mm}$ voxel resolution of the CT dataset. Performance of the rigid registration was visually assessed before continuing. After rigid registration, each resulting MRI dataset was non-rigidly registered to the $\mathrm{CT}$ dataset. The deformable registration method was based on a cubic B-spline free-deformation model using a normalised mutual information metric from the non-commercial open source software (NiftyReg version 1.3.9) ${ }^{36}$. The registration was performed in 4 iterations with final grid spacing of $25 \mathrm{~mm}$. In this implementation, all control points were optimised and 
interpolation performed in 3D across the whole image at each step. The accuracy of the registration algorithm was within half a pixel.

\section{II.D.2. Gradient and $B_{0}$ distortion components:}

Non-rigid image registration resulted in the determination of the total geometric distortion (dist_total). Following a previously reported method ${ }^{33,34}$, image acquisition was repeated with the frequency encode direction reversed. The frequency encoding direction is subject to geometric errors from both $B_{0}$ inhomogenity and gradient nonlinearity:

$$
y_{1}=y+\frac{\Delta B_{0}(x, y, z)}{G_{y}}+\frac{\Delta B_{G y}(x, y, z)}{G_{y}}
$$

where $\mathrm{y}_{1}$ is the distorted location, $\mathrm{y}$ is the true position, $\Delta \mathrm{B}_{0}$ is the distortion contribution from $\mathrm{B}_{0}$ inhomogeneity, $\mathrm{Gy}$ is the frequency encoding strength and $\Delta \mathrm{B}_{\mathrm{Gy}}$ is the distortion contributions from gradient nonlinearities in the frequency encoding direction. Analysis on images with reversed frequency encoding directions can separate these components. For this study, the deformation field obtained by registering the MR images of opposing frequency encode directions was halved to give the distortion due to $B_{0}$ inhomogeneity (dist_ $B_{0}$ ). This was then subtracted from the total deformation field obtained in the MR-CT registration, giving the residual distortions, due to the gradient nonlinearities (dist_Grad) (Fig. 3).

\section{RESULTS}

\section{III.A. Standard static acquisition sequences}

Distortions due to residual gradient nonlinearities and $\mathrm{B}_{0}$ inhomogeneities are separated in Fig. 4

255 for the standard SE sequence acquired with $2 \mathrm{~mm}$ slice thickness (3D correction algorithm applied). The vector fields are thresholded to highlight regions within the imaging FOV where distortions were greater than $2 \mathrm{~mm}$. The vectors indicate the total distortion in all directions ( $x, y$ and $z)$. Distortions resulting from residual gradient nonlinearities are greater than those due to the $B_{0}$ inhomogeneity. The dist_Grad were 
greatest at the superior and inferior regions of the image with dist_ $B_{0}$ largest at the extreme regions of the axial FOV.

Table 3 indicates the overall geometric performance of each sequence (dist_total), accounting for variations in scan length due to sequence capabilities. The table includes results for 2D and 3D corrected images, the percentage of the phantom length scanned and differences in the image length acquired per second, based on the acquisition time of each sequence. Images with the retrospective 3D correction applied had an improved geometrical performance.

Maximum distortions for the $2 \mathrm{~mm} \mathrm{SE}, 3 \mathrm{~mm}$ SE and CISS sequences were $7.9 \mathrm{~mm}, 9.8 \mathrm{~mm}$ and 7.6 $\mathrm{mm}$ respectively with the $2 \mathrm{D}$ distortion algorithm applied. With the 3D correction algorithm applied retrospectively, these were reduced to $4.08 \mathrm{~mm}, 4.4 \mathrm{~mm}$ and $4.9 \mathrm{~mm}$. These maximum distortions occurred in the most superior and inferior regions of the image.

We define the convergence region of the registration algorithm as the region where the accuracy of the registration algorithm is better than 1 pixel. This region was found to be within a length of $\pm 119 \mathrm{~mm}$ and an axial radial distance of $r=180 \mathrm{~mm}$ from the centre of the scanner. The magnitude of distortion was accurately measured within this region, even when the spatial displacement greatly exceeded the $2 \mathrm{~mm}$ tolerance limit. Beyond this volume, extreme distortion resulted in some capsules no longer being visible in the image. As such, distortion measurements beyond this region could not be reliably measured but can be assumed to be much greater than $2 \mathrm{~mm}$. Black rings began appearing beyond this point in the images with the 3D correction algorithm applied in regions of severe distortion or where capsules were not observable in the $2 \mathrm{D}$ corrected images.

a)

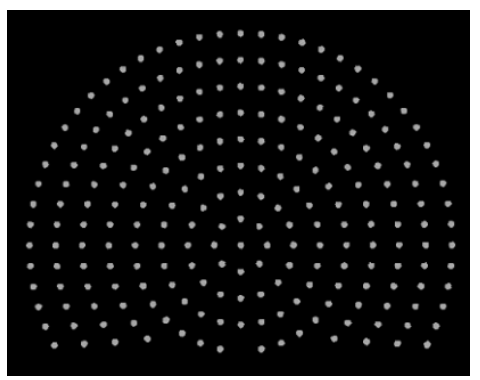

b)

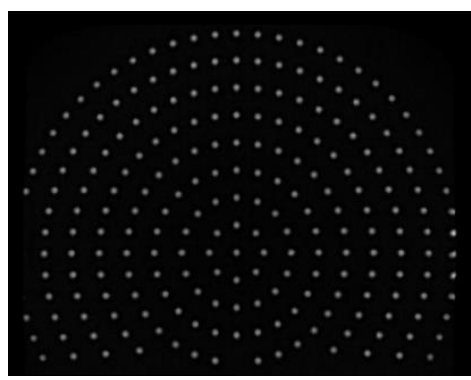

c)

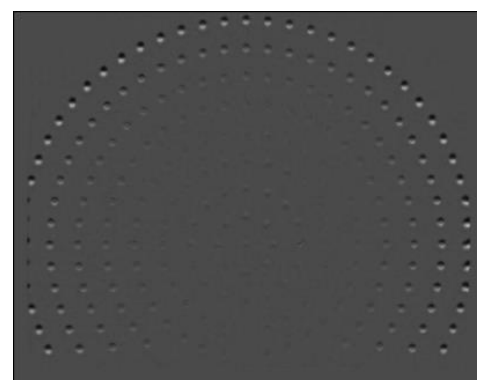



subtraction of two MR images with frequency encode direction reversed.

a)

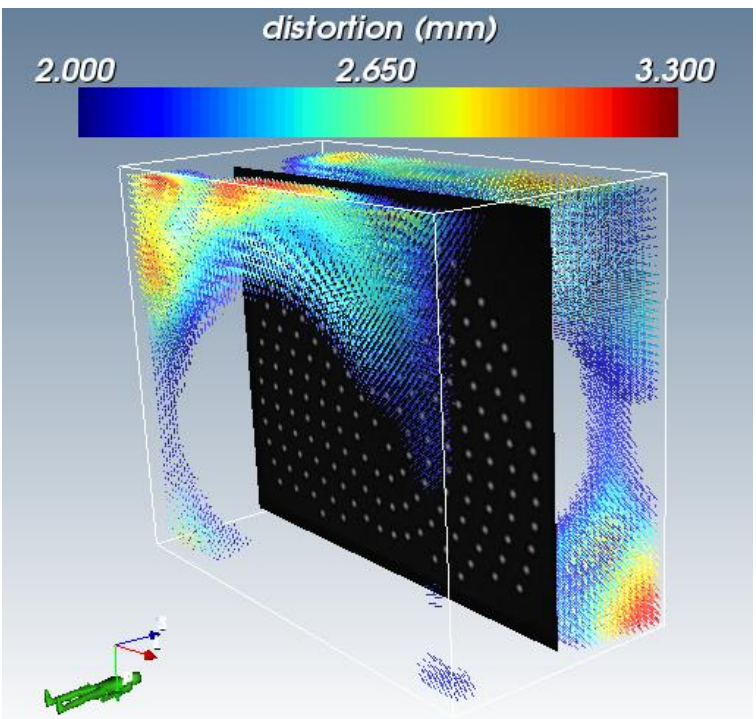

b)

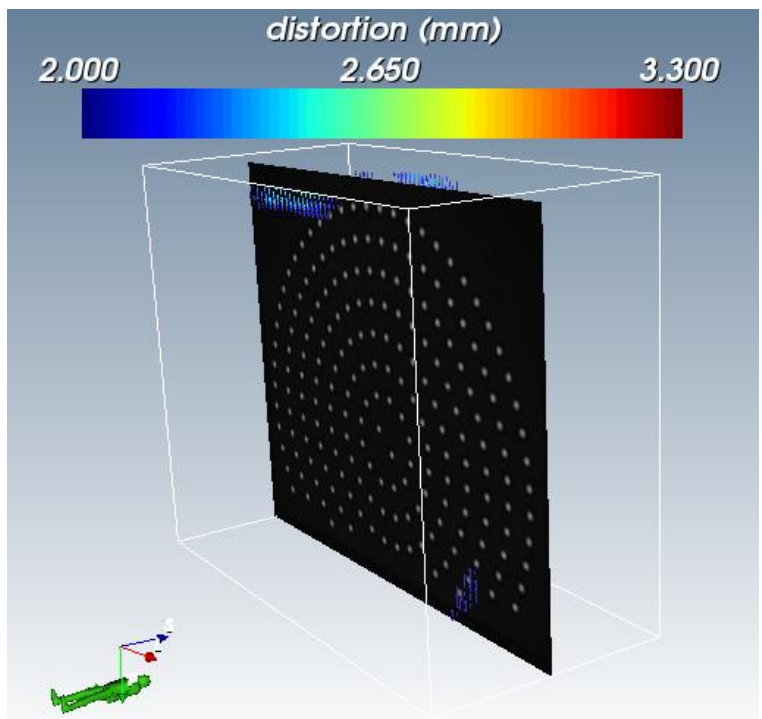

Fig. 4: Vector maps indicating regions of distortion $\geq 2 \mathrm{~mm}$ for the a) dist_Grad and b) dist_Bo components of distortion for the standard 2D SE acquisition sequence with $2 \mathrm{~mm}$ slice thickness with the 3D correction algorithm applied. Visualisation provided by the Simple Medical Imaging Library Interface (SMILI) http://aehrc.com/research/biomedical-imaging/software-solutions ${ }^{37}$

Table 3: Geometric performance of the acquisition sequences

\begin{tabular}{ccccc}
\hline Sequence & $\begin{array}{c}\text { \% phantom } \\
\text { imaged }\end{array}$ & $\begin{array}{c}\text { Distance from } \\
\text { isocenter where } \\
\text { distortion } \leq 2 \mathrm{~mm}\end{array}$ & $\begin{array}{c}\text { Nominal acquisition } \\
\text { distortion }<2 \mathrm{~mm}\end{array}$ & $\begin{array}{c}\text { time* } \\
\text { (minutes : seconds) }\end{array}$ \\
\hline \hline (3D corr) & 49 & $98 \mathrm{~mm}$ & 29 & $29: 56$ \\
\hline (3D corr) & 72 & $152 \mathrm{~mm}$ & 44 & $20: 22$ \\
\hline CISS (2D corr) & 69 & $154 \mathrm{~mm}$ & 30 & $13: 30$ \\
\hline
\end{tabular}




\begin{tabular}{ccccc}
\hline (3D corr) & & $148 \mathrm{~mm}$ & 51 & \\
\hline Spoiled GRE (Non & 71 & $147 \mathrm{~mm}$ & 47 & \\
TimCT) & 100 & $140 \mathrm{~mm}$ & 87 & \\
\hline Spoiled GRE & & 51 & $5: 26$ \\
$($ TimCT 1.1 mm/s) & 100 & $55 \mathrm{~mm}$ & 61 & \\
\hline Spoiled GRE & & & \\
\hline
\end{tabular}

* Nominal acquisition time $=$ time required to image whole phantom

\section{III.B. TimCT}

Vector field maps for the total distortion observed for the spoiled GRE sequence acquired with a static table and TimCT with table speeds of $1.1 \mathrm{~mm} / \mathrm{s}$ and $2 \mathrm{~mm} / \mathrm{s}$ are shown in Fig. 5 . The longer scan length imaged with TimCT is evident when compared to the static table acquisition. Distortion patterns for TimCT scans were more consistent through the phantom volume compared to the static table where a large reduction in geometric integrity was observed at the edge of the field where the through plane distortion was worse. Maximum distortions (dist_total) were $6.1 \mathrm{~mm}$ (71\% phantom volume imaged), 4.4 $\mathrm{mm}$ and $5.8 \mathrm{~mm}$ for the static, $1.1 \mathrm{~mm} / \mathrm{s}$ and $2 \mathrm{~mm} / \mathrm{s}$ table speeds respectively.

Figure 6 shows the percentage of points within a defined phantom volume where distortions were observed to be greater than $2 \mathrm{~mm}$ for the static table, TimCT $1.1 \mathrm{~mm} / \mathrm{s}$ and TimCT $2 \mathrm{~mm} / \mathrm{s}$ scans respectively. The volumes were defined from the centre of the phantom, with varying through plane lengths $( \pm z)$ and radial distances in the axial plane $(r)$. All measurements were reported with respect to the phantom centre as opposed to scanner isocenter since this provided a consistent reference point for static and moving table acquisitions. The maximum distortion observed is reduced when TimCT is utilised. Table 3 compares the spoiled GRE sequence investigated for TimCT purposes to the standard static acquisition sequences. 
Figure 7 compares the geometric performance of the spoiled GRE without TimCT compared to TimCT with a table speed of $1.1 \mathrm{~mm} / \mathrm{s}$ over different radial distances and scan lengths from the scanner centre. It shows the volume percentage comparison in which distortions are less than $2 \mathrm{~mm}$ within the volume in question. Values greater than 1 indicate the TimCT sequence performed better geometrically. As was found for the standard static sequence acquisitions, beyond the convergence region of a length of $\pm 119 \mathrm{~mm}$ and radial distance of $r=180 \mathrm{~mm}$ from the phantom centre on the static spoiled GRE, grid points were either not visible in the image or the distortions were too great for the registration algorithm to perform accurately beyond this point. The registration algorithm was not found to fail for the TimCT images. 
a)

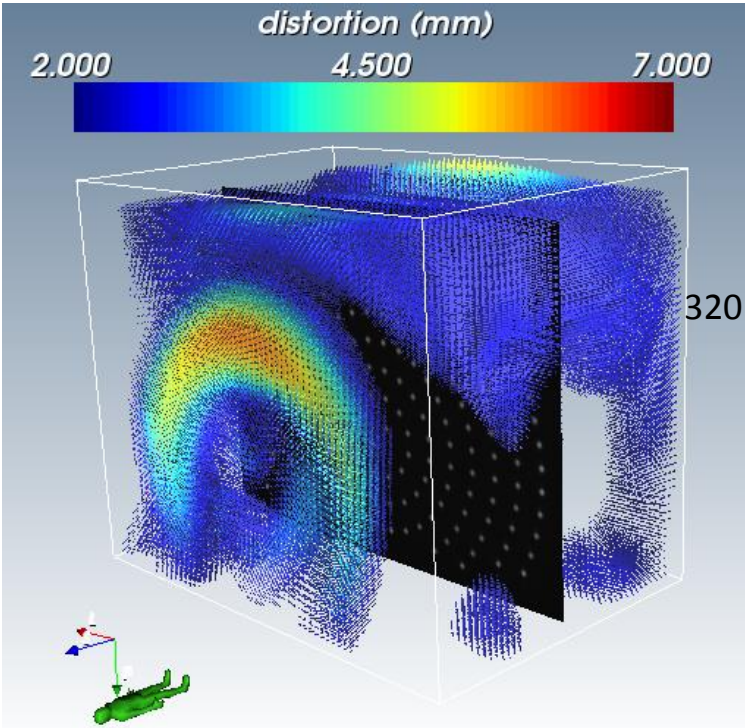

b)

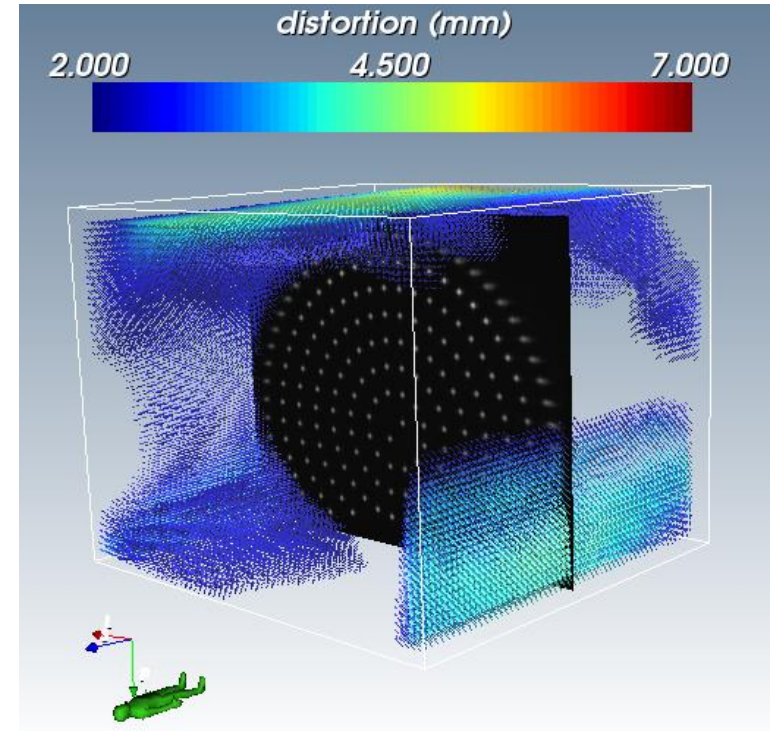

c)

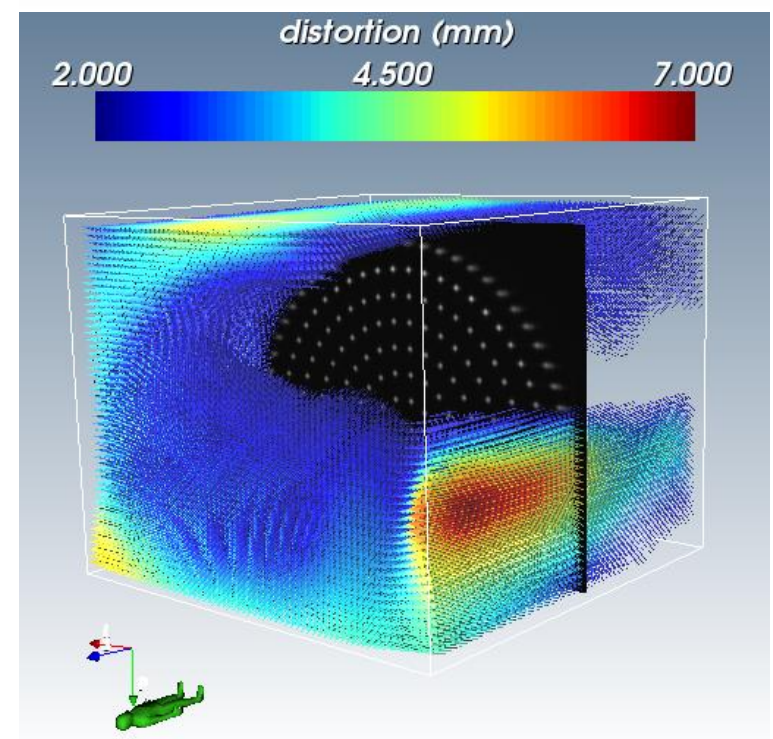

Fig. 5: Vector maps indicating regions where total distortion $\geq 2 \mathrm{~mm}$ for the spoiled GRE sequence acquired with table speeds of a) $0 \mathrm{~mm} / \mathrm{s}, b) 1.1$ $\mathrm{mm} / \mathrm{s}$ and c) $2 \mathrm{~mm} / \mathrm{s}$ 
a)

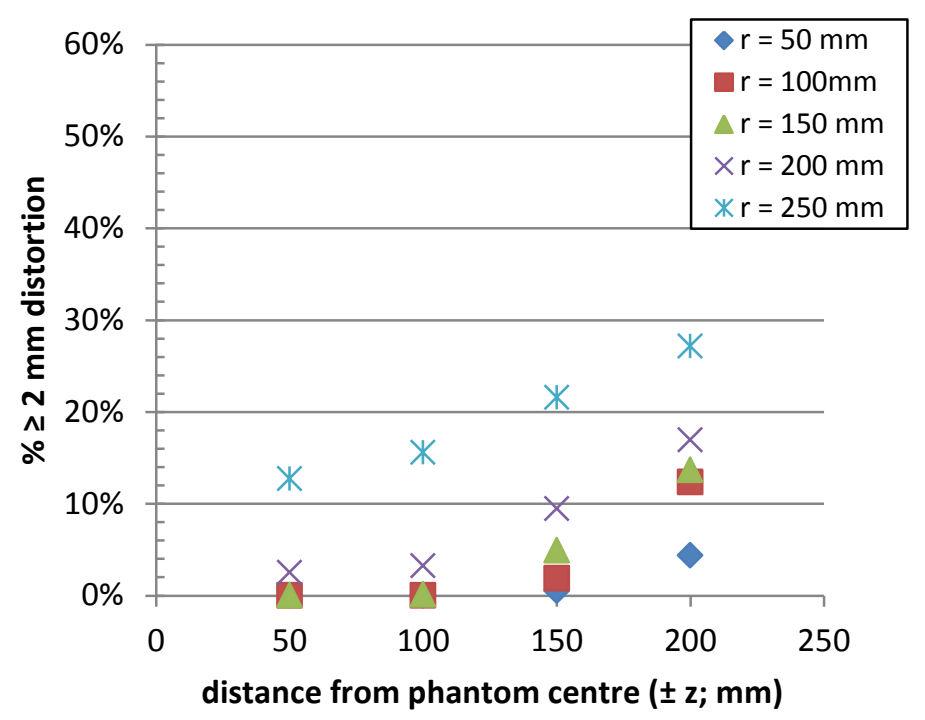

b)

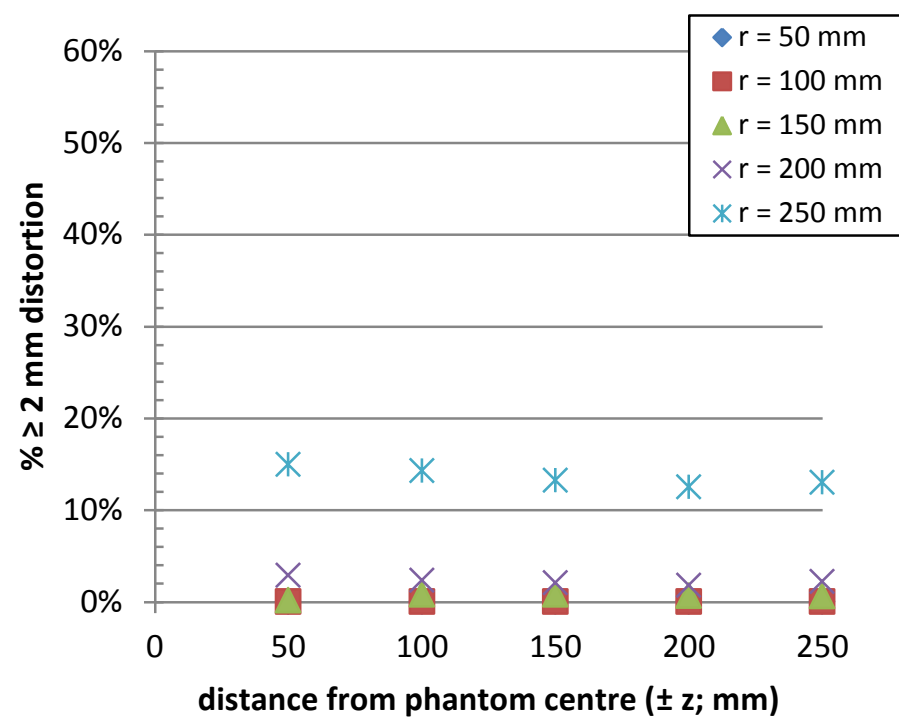

c)

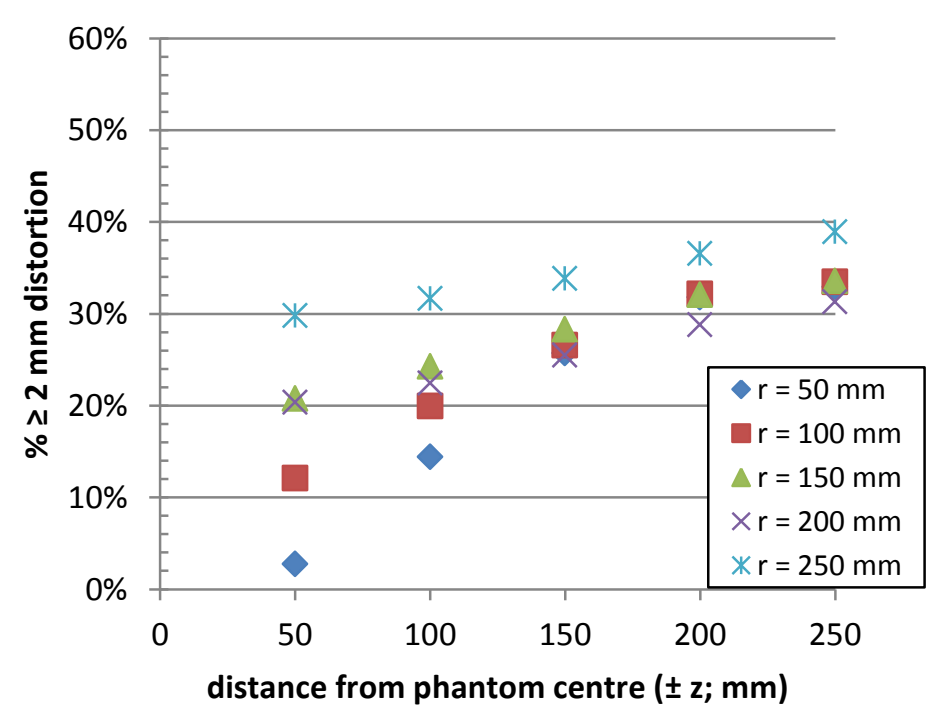

Fig. 6: Normalised percentage of points

within the imaged volume at a given

distance from the centre of the

phantom $( \pm z)$ for a given axial radial

distance from the centre of the scanner

(r) where the distortion is greater than

or equal to $2 \mathrm{~mm}$ for the spoiled GRE

330 with a) Non TimCT, b) TimCT at 1.1

$\mathrm{mm} / \mathrm{s}$ table speed and, c) TimCT at 2

$\mathrm{mm} / \mathrm{s}$ table speed 


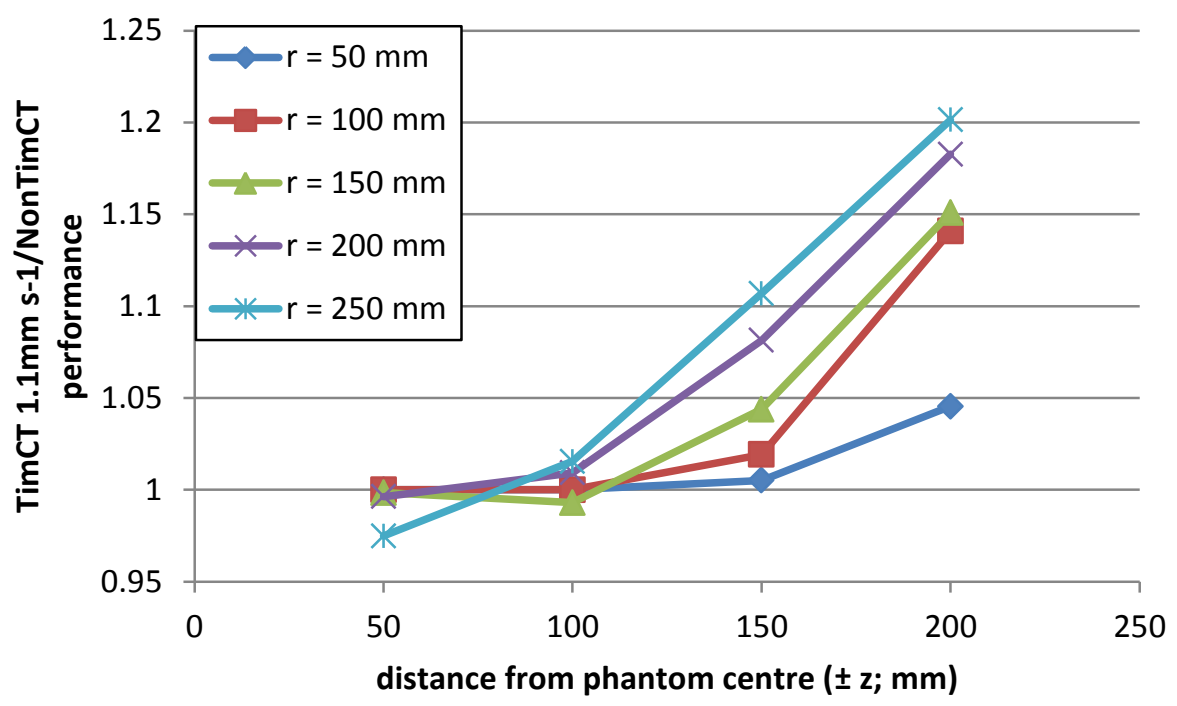

335

Fig. 7: Ratio of the spoiled GRE regions where distortion $<2 \mathrm{~mm}$ for images acquired with TimCT at 1.1 $\mathrm{mm} / \mathrm{s}$ and with a static table (TimCT/Non-TimCT). Values greater than 1 indicate the TimCT sequence performed better geometrically

\section{DISCUSSION}

The developed phantom enables measurements of MRI distortion across the full FOV as required for RTP, where the patient anatomy is required for dose calculations. The axial size of the phantom should encompass the size of patients which would be suited for MR scanning, allowing for additional placement of surface coils over the patient/phantom. The phantom length makes it suitable for imaging over various scan lengths without the need for physical relocation and re-scanning. The extended length also allows assessment of techniques such as the TimCT moving table option. While the phantom weighed $60 \mathrm{~kg}$, a water phantom of similar volume would have resulted in a final weight of over $85 \mathrm{~kg}$. When measuring system distortion it is important to reduce distortion contributions from the phantom itself. This was achieved by carefully considering the design of the phantom to achieve a tight fit of elongated capsules to reduce susceptibility from air and the capsules themselves. The dimension of the capsules provides a 
sufficient signal point source while still enabling assessment of through plane distortion (unlike rods for example).

The distortion distributions observed in Fig. 4 highlight the optimisation characteristics of the scanner utilised. When looking at the gradient component of distortion, the distortion magnitude is worse at the superior-inferior edges of the FOV where the gradient field linearity declines due to the short bore size. The $B_{0}$ field components show that this effect is worse axially around the bore edges as opposed to the through plane edges. This highlights the cylindrical nature of the magnet used in this new scanner and emphasises the need for QA on each specific system. It should be noted that $B_{0}$ inhomogeneity is a more crucial component when imaging certain patient anatomy (e.g. sinuses within the head), creating regional variations on top of the system effects described here. In clinical acquisitions appropriate steps should be taken to address this including further increasing the bandwidth, more localised volumetric shimming and even considering $B_{0}$ correction. Choice of appropriate MRI protocols ${ }^{7,38}$ within a department is an important part of this process. Comparison of the static image acquisitions in table 3 highlights the difference in 2D and 3D vendor correction algorithm application. During image acquisition, the SE and CISS sequences only permitted 2D correction application while the 3D correction could be applied to the spoiled GRE. For the SE and CISS sequences, retrospective 3D correction could be performed. This highlights a practical limitation that may be encountered during imaging as well as emphasising the importance of knowing the capabilities of each system, both on and offline. Since the 2D correction algorithm does not consider the slice selection direction, through plane distortions are not taken into account. As expected, the geometric performance of sequences acquired with the 2D algorithm applied was poorer than the 3D corrected images in which distortion is considered in all 3 axes. This resulted in an improvement of the percentage of the phantom volume where distortions were within $2 \mathrm{~mm}$ of $15 \%, 24 \%$ and $19 \%$ (absolute percentage coverage) for the $3752 \mathrm{~mm} \mathrm{SE}, 3 \mathrm{~mm} \mathrm{SE}$ and CISS sequences respectively. Distortions greater than $2 \mathrm{~mm}$ were not observed within a radial distance of $148 \mathrm{~mm}$ from isocenter. For radiotherapy purposes, the highest order correction algorithm available should be applied. Restrictions in scanner and sequence capabilities in applying these 
corrections indicate more potential improvements could be made towards MRI scanning options for the purposes of RTP.

The moving table option offered with TimCT acquisition provided a significant increase to the field length that can be imaged compared to static table acquisition (table 3; Figs. 5 and 6). The vendor quotes the TimCT option allows for imaging with field lengths of $205 \mathrm{~cm}$. It was tested to $50 \mathrm{~cm}$ in this study which encompassed the length of the phantom and exceeded the maximum scan length limits of static table acquisition.

Of the sequences investigated, TimCT acquisition with a table speed of $1.1 \mathrm{~mm} / \mathrm{s}$ performed the best geometrically. However this came at the cost of time. Acquisition time for the TimCT $1.1 \mathrm{~mm} / \mathrm{s}$ was 9 minutes, 30 seconds, 3.5 times greater than that of the static table acquisition for the same spoiled GRE sequence ( 2 minutes, 41 seconds to image the whole phantom). While that resulted in increased image scan length and distortion improvement at superior and inferior regions of the image, the additional scan time could lead to increased patient movement and associated artifacts within the image, potentially reducing its benefit for RTP. Time variations are also subject to the desired imaging sequence. For example, TimCT at $1.1 \mathrm{~mm} / \mathrm{s}$ with the spoiled GRE resulted in an improved acquisition time when compared to the static table SE sequences (14 minutes, 40 seconds), the latter with scan lengths of only $47-68 \%$ that of the spoiled TimCT GRE. movement. This is evident when comparing the $1.1 \mathrm{~mm} / \mathrm{s}$ and $2 \mathrm{~mm} / \mathrm{s}$ table speeds (Fig. $5 \mathrm{~b}, \mathrm{c}$; Fig $6 \mathrm{~b}, \mathrm{c}$ ). Through plane movement results in blurring of the capsules and an increase in the severity of the gradient nonlinearity distortions across all 3 imaging planes. This effect worsens with increasing distance from the isocenter of the scanner due to the increased gradient nonlinearities, as discussed for blood vessel imaging in MRA by Polzin et al. ${ }^{18}$.

Table movement amplifies the system distortions and manifests as blurring within the image. Since these distortions are larger at the outer edges of the FOV, blurring is worse in these regions with the effect minimal in the central FOV. With TimCT acquired at $1.1 \mathrm{~mm} / \mathrm{s}$, blurring resulted in an increase in apparent 
capsule volume of $43 \pm 5 \%$ ( \pm 2 standard deviations) at $r=225 \mathrm{~mm}$ from scanner centre and $95 \pm 8 \%$ at $r=$ $240 \mathrm{~mm}$. Blurring increased with table speed with the $2 \mathrm{~mm} / \mathrm{s}$ TimCT average capsule volume increasing by $93 \pm 1 \%$ and $170 \pm 5 \%$ at $r=225$ and $r=240 \mathrm{~mm}$, respectively. Increasing the table speed from $1.1 \mathrm{~mm} / \mathrm{s}$ to $2 \mathrm{~mm} / \mathrm{s}$ resulted in a $26 \%$ increase in the percentage of grid points experiencing distortions greater than $2 \mathrm{~mm}$. While TimCT at $2 \mathrm{~mm} / \mathrm{s}$ permitted a reduction in maximum distortion and a more regular distortion pattern compared to the static table acquisitions, it performed worst in the distance from isocenter where distortions greater than $2 \mathrm{~mm}$ became observable. Distortions became greater than $2 \mathrm{~mm}$ beyond a radial distance of $55 \mathrm{~mm}$ from phantom centre. This corresponded to the maximum distance from scanner isocenter that an image could be acquired at that speed.

Figure 7 indicated regions for the best geometric performance between the static and $1.1 \mathrm{~mm} / \mathrm{s}$ TimCT image acquisitions. When imaging within a small radius from isocenter with a short scan length, the two techniques perform similarly, with the Non TimCT acquisition performing slightly better for a larger scan length. As the scan length increased beyond $\pm 100 \mathrm{~mm}$ from phantom centre, the TimCT sequence was found to perform best for all radial distances investigated. This is dependent on the table speed. When the table speed was increased to $2 \mathrm{~mm} / \mathrm{s}$, the static sequence performed geometrically better, though it was not able to image the whole phantom. This information is useful when determining what acquisition technique to use for the purposes of RTP, given the anatomy of interest, the FOV required and the role of the MRI data. For example for centrally located anatomy, such as brain, head \& neck or when deliberately using a small FOV (e.g. prostate) to subsequently register to $\mathrm{CT}$, static acquisition may be sufficient. As indicated in figure 6 , if imaging over a distance of $\pm 100 \mathrm{~mm}$ from the phantom centre, the static image and TimCT at $1 \mathrm{~mm} / \mathrm{s}$ perform very similarly, with distortions greater than $2 \mathrm{~mm}$ increasing beyond a radial distance of $200 \mathrm{~mm}$ from the scanner centre. In other instances where extended z coverage is warranted or anatomically suited (e.g. peripheral sites, lung etc.) then a moving table strategy may be important. Figure 6 highlights the potential use of TimCT for this purpose. At $1 \mathrm{~mm} / \mathrm{s}$, the percentage of points experiencing more than $2 \mathrm{~mm}$ distortion remaining constant over the whole phantom volume, compared to the static case where the whole volume could not be imaged and the distortion increased with increasing distance 
from the phantom centre. Although TimCT is currently a vendor specific implementation, this work has shown advantages in repositioning anatomy rather than simply extending the image volume which should be considered on other systems using stepping table variations. This paper served to highlight the geometrical variations that these techniques manifest and emphasises the importance of understanding the limitations of MRI acquisitions. analysed however, with capsules towards the outer edge of the phantom in axial slices becoming severely blurred and displaced. Some of these points were no longer observable in the image and the analysis process would not have been able to account for the loss of information. Increasing the table speed was done at the expense of the slab length imaged as the table moved through the scanner. Faster table speeds were therefore susceptible to additional blurring due to the movement as well as increased gradient nonlinearity effects due to an imaging area extending further from isocenter.

One of the limitations of the study was the performance of the registration algorithm at high distortion regions at the FOV edges in the static acquisitions. The registration performed well in central regions of the phantom where no grid point information was lost. Outside the convergence region of the registration algorithm, the image contrast is not sufficient and thus the algorithm merely extrapolates the transformation. This was apparent from visual inspection. Distortion values greater than $2 \mathrm{~mm}$ were assumed for regions of the phantom that could not be imaged with static acquisitions and the regions in which the registration algorithm failed. Because the distortion patterns were more regular throughout the whole imaging volume for the TimCT scans and there was no field limitations resulting in a loss of capsule visualisation, the registration algorithm was not found to fail for these images.

The ability to change imaging parameters from the default sequence setting for TimCT was more restricted than the static spoiled GRE acquisition option. Of particular note for this study were the slice thickness options available and the restrictions this put on table speed options. As a result, the slice thickness of $5 \mathrm{~mm}$ investigated for this sequence was larger than the $2 \mathrm{~mm}$ generally used for treatment planning on CT. TimCT was also limited to 2 acquisition sequences, the spoiled GRE and a single shot turbo 
spin echo (TSE) sequence. The TSE sequence was scanned during preliminary data collection however the SNR on the phantom images was too low for the purposes of image analysis.

Static or moving table MRI acquisition for RTP would need to be assessed based on the application. Both techniques could be useful in providing supplementary information to planning CTs. Static images can provide the geometric information required, particularly over anatomical regions extending over a shorter scan length. In some cases, TimCT would be required to ensure adequate scan length coverage, particularly if dealing with long OARs or target volumes. The same argument could be used for their application in MRonly planning.

\section{CONCLUSION}

A novel phantom design for measuring MRI distortion over a large 3D volume of the scanner has been presented, which permits the mapping of geometrical accuracy across the whole imaging volume as required for RTP. Measurements have been made with both static and moving table acquisitions to demonstrate variations in the achievable geometric accuracy. The moving table technique (TimCT) was shown to extend the distortion free volume of the phantom by continuously acquiring data from a small section around the scanner isocenter. There are a number of limitations to this procedure including choice of available pulse sequence and effects of increasing table speed. However, this work illustrates the nature of MRI distortions, demonstrating geometric variations that can occur between image acquisition techniques (as opposed to sequence variations) which is not well known in the RT community. It is important to fully characterise an individual MRI system and use this knowledge to select the most suitable imaging protocol for radiotherapy treatment planning.

\section{ACKNOWLEDGEMENTS}

The authors would like to acknowledge Robba Rai and Ewa Juresic for their knowledge and assistance with the MRI scanning of the phantom. Thank you to Jonathan Knott and Craig Davis for the 
phantom design drawing and construction respectively. Finally thank you to Shekhar Chandra for additional software help during the image analysis process.

1. P. Metcalfe, G. P. Liney, L. Holloway, A. Walker, M. Barton, G. P. Delaney, S. Vinod and W. Tomé, "The Potential for an Enhanced Role for MRI in Radiation-Therapy Treatment Planning," Technology in Cancer Research \& Treatment 12, (5), 429-446 (2013).

2. D. Wang, W. Strugnell, G. Cowin, D. M. Doddrell and R. Slaughter, "Geometric distortion in clinical MRI systems: Part I: evaluation using a 3D phantom," Magnetic Resonance Imaging 22, (9), 490 1211-1221 (2004).

3. A. Walker, G. Liney, P. Metcalfe and L. Holloway, "MRI distortion: considerations for MRI based radiotherapy treatment planning," Australas Phys Eng Sci Med 37, (1), 103-113 (2014).

4. D. Mah, M. Steckner, E. Palacio, R. Mitra, T. Richardson and G. E. Hanks, "Characteristics and quality assurance of a dedicated open $0.23 \mathrm{~T}$ MRI for radiation therapy simulation," Medical Physics 29, (11), 2541-2547 (2002).

5. G. J. Kutcher, L. Coia, M. Gillin, W. F. Hanson, S. Leibel, R. J. Morton, J. R. Palta, J. A. Purdy, L. E. Reinstein, G. K. Svensson and et al., "Comprehensive QA for radiation oncology: report of AAPM Radiation Therapy Committee Task Group 40," Med Phys 21, (4), 581-618 (1994).

6. G. P. Liney, S. C. Owen, A. K. E. Beaumont, V. R. Lazar, D. J. Manton and A. W. Beavis, 500 "Commissioning of a new wide-bore MRI scanner for radiotherapy planning of head and neck cancer," The British Journal of Radiology 86, (1027), 20130150 (2013).

7. G. P. Liney and M. A. Moerland, "Magnetic Resonance Imaging Acquisition Techniques for Radiotherapy Planning," Seminars in Radiation Oncology 24, (3), 160-168 (2014).

8. $\quad$ AAPM, Report No. 100, 2010.

505 9. A. Landi, R. Marina, C. DeGrandi, A. Crespi, G. Montanari, E. P. Sganzerla and S. M. Gaini, "Accuracy of Stereotactic Localisation with Magnetic Resonance Compared to CT Scan: Experimental Findings," Acta Neurochir (Wien) 143, (6), 593-601 (2001).

10. F. J. Prott, U. Haverkamp, N. Willich, A. Resch, U. Stöber and R. Pötter, "Comparison of imaging accuracy at different MRI units based on phantom measurements," Radiotherapy and 510 Oncology 37, (3), 221-224 (1995).

11. S. P. M. a. R. Crijns, B.W. and Lagendijk, J.J.W., "Real-time correction of magnetic field inhomogeneity-induced image distortions for MRI-guided conventional and proton radiotherapy," Physics in Medicine and Biology 56, (1), 289-297 (2011).

12. K. Engelhard, H. P. Hollenbach, K. Wohlfart, E. Imhoff and F. A. Fellner, "Comparison of 515 whole-body MRI with automatic moving table technique and bone scintigraphy for screening for bone metastases in patients with breast cancer," Eur Radiol 14, (1), 99-105 (2004).

13. E. Paulson, R. Prost and X. Li, "TU-E-BRC-05: "Step and Shoot MRI:" a Simple Acquisition Method to Reduce Gradient Nonlinearity-Induced Geometric Distortions for Radiation Treatment Planning," Medical Physics 38, (6), 3770-3770 (2011).

520 14. H. P. Fautz and S. A. R. Kannengiesser, "Sliding multislice (SMS): A new technique for minimum FOV usage in axial continuously moving-table acquisitions," Magnetic Resonance in Medicine 55, (2), 363-370 (2006).

15. D. G. Kruger, S. J. Riederer, R. C. Grimm and P. J. Rossman, "Continuously moving table data acquisition method for long FOV contrast-enhanced MRA and whole-body MRI," Magnetic Resonance in Medicine 47, (2), 224-231 (2002). 
16. P. Börnert and B. Aldefeld, "Principles of whole-body continuously-moving-table MRI," Journal of Magnetic Resonance Imaging 28, (1), 1-12 (2008).

17. J. Schaefer and H.-P. Schlemmer, "Total-body MR-imaging in oncology," Eur Radiol 16, (9), 2000-2015 (2006).

530 18. J. A. Polzin, D. G. Kruger, D. H. Gurr, J. H. Brittain and S. J. Riederer, "Correction for gradient nonlinearity in continuously moving table MR imaging," Magnetic Resonance in Medicine 52, (1), 181-187 (2004).

19. A. Janke, H. Zhao, G. J. Cowin, G. J. Galloway and D. M. Doddrell, "Use of spherical harmonic deconvolution methods to compensate for nonlinear gradient effects on MRI images," Magnetic Resonance in Medicine 52, (1), 115-122 (2004).

20. P. Dammann, O. Kraff, S. Maderwald, E. R. Gizewski, M. Ladd, T. Gasser and U. Sure, "Evaluation of geometrical distortion in a head-sized phantom at ultra-high-field MRI (7 Tesla) for image guided applications in neurosurgery, presented at the The EANS Annual Meeting, Groningen, The Netherlands, 2010.

540 21. J. L. Gunter, M. A. Bernstein, B. J. Borowski, C. P. Ward, P. J. Britson, J. P. Felmlee, N. Schuff, M. Weiner and C. R. Jack, "Measurement of MRI scanner performance with the ADNI phantom," Medical Physics 36, (6), 2193-2205 (2009).

22. B. Zhang, D. MacFadden, A. Z. Damyanovich, M. Rieker, J. Stainsby, M. Bernstein, D. A. Jaffray, D. Mikulis and C. Menard, "Development of a geometrically accurate imaging protocol at 3

545 Tesla MRI for stereotactic radiosurgery treatment planning," Physics in Medicine and Biology 55, (1), 6601-6615 (2010).

23. C. Hong, D.-H. Lee and B. S. Han, "Characteristics of geometric distortion correction with increasing field-of-view in open-configuration MRI," Magnetic Resonance Imaging 32, (6), 786-790 (2014).

$55024 . \quad$ r. C. Orth, P. Sinha, E. L. Madsen, G. Frank, F. R. Korosec, T. R. Mackie and M. Mehta, "Development of a Unique Phantom to Assess the Geometric Accuracy of Magnetic Resonance Imaging for Stereotactic Localization," Neurosurgery 45, (6) (1999).

25. S. F. Tanner, D. J. Finnigan, V. S. Khoo, P. Mayles, D. P. Dearnaley and M. O. Leach, "Radiotherapy planning of the pelvis using distortion corrected MR images: the removal of system distortions," Phys Med Biol 45, (8), 2117-2132 (2000).

26. S. J. Doran, L. Charles-Edwards, S. A. Reinsberg and M. O. Leach, "A complete distortion correction for MR images: I. Gradient warp correction," Physics in Medicine and Biology 50, (7), 1343 (2005).

27. Z. Caramanos, V. S. Fonov, S. J. Francis, S. Narayanan, G. B. Pike, D. L. Collins and D. L.

560 Arnold, "Gradient distortions in MRI: Characterizing and correcting for their effects on SIENAgenerated measures of brain volume change," Neurolmage 49, (2), 1601-1611 (2010).

28. V. S. Fonov, A. Janke, Z. Caramanos, D. L. Arnold, S. Narayanan, G. B. Pike and D. L. Collins, "Improved precision in the measurement of longitudinal global and regional volumetric changes via a novel MRI gradient distortion characterization and correction technique," Medical Imaging and Augmented Reality, 324-333 (2010).

29. H.-H. Chen, R. D. Boykin, G. D. Clarke, J.-H. T. G. J. W. Roby and III, "Routine testing of magnetic field homogeneity on clinical MRI systems," Medical Physics 33, (11), 4299-4306 (2006).

30. D. Wang and D. M. Doddrell, "Method for a detailed measurement of image intensity nonuniformity in magnetic resonance imaging," Medical Physics 32, (4), 952-960 (2005).

570 31. T. Stanescu, K. Wachowicz and D. A. Jaffray, "Characterization of tissue magnetic susceptibility-induced distortions for MRIgRT," Med Phys 39, (12), 7185-7193 (2012). 
32. R. Bhagwandien, M. A. Moerland, C. J. G. Bakker, R. Beersma and J. J. W. Lagendijk, "Numerical analysis of the magnetic field for arbitrary magnetic susceptibility distributions in 3D," Magnetic Resonance Imaging 12, (1), 101-107 (1994).

575 33. L. N. Baldwin, K. Wachowicz and B. G. Fallone, "A two-step scheme for distortion rectification of magnetic resonance images," Medical Physics 36, (9), 3917-3926 (2009).

34. M. A. Moerland, R. Beersma, R. Bhagwandien, H. K. Wijrdeman and C. J. G. Bakker, "Analysis and correction of geometric distortions in $1.5 \mathrm{~T}$ magnetic resonance images for use in radiotherapy treatment planning," Physics in Medicine and Biology 40, (10), 1651 (1995).

580 35. D. Rivest-Hénault, N. Dowson, P. Greer and J. Dowling, "Inverse-consistent rigid registration of $\mathrm{CT}$ and MR for MR-based planning and adaptive prostate radiation therapy," Journal of Physics: Conference Series 489, (1), 012039 (2014).

36. M. Modat, G. R. Ridgway, Z. A. Taylor, M. Lehmann, J. Barnes, D. J. Hawkes, N. C. Fox and S. Ourselin, "Fast free-form deformation using graphics processing units," Computer methods and 585 programs in biomedicine 98, (3), 278-284 (2010).

37. S. S. Chandra, Y. Xia, C. Engstrom, S. Crozier, R. Schwarz and J. Fripp, "Focused shape models for hip joint segmentation in 3D magnetic resonance images," Medical Image Analysis 18, (3), 567-578 (2014).

38. J. N. H. Brunt, "Computed Tomography-Magnetic Resonance Image Registration in Radiotherapy Treatment Planning," Clinical Oncology 22, (8), 688-697 (2010). 